\title{
Electrochemical Immunosensors for Celiac Disease Detection
}

\author{
Daniel Martín-Yerga, Agustín Costa-García* \\ Nanobioanalysis group, Department of Analytical and Physical Chemistry, University of Oviedo, Oviedo (Spain) \\ *Corresponding author: costa@uniovi.es
}

Received September 10, 2014; Revised September 15, 2014; Accepted October 04, 2014

\begin{abstract}
Cite This Article: Daniel Martín-Yerga, and Agustín Costa-García, "Electrochemical Immunosensors for Celiac Disease Detection." International Journal of Celiac Disease, vol. 2, no. 4 (2014): 139-141. doi: 10.12691/ijcd-2-4-1.
\end{abstract}

\section{Introduction}

Unfortunately, a high percentage of people with celiac disease $(C D)$ is not yet diagnosed. The disease could begin at any age and the different produced symptoms may complicate the diagnosis, delay the treatment and worsening the living conditions of the patient. Therefore, early diagnosis of the disease when patients have few or not symptoms is really important and can help to improve the patients' life quality. In these cases, a small bowel biopsy, which is the most accurate detection method, is completely ruled out, and blood tests should be key to the early diagnosis.

Although, biopsy is still the most common method for confirming the CD diagnosis, the detection of diseasespecific autoantibodies strongly support this diagnosis. The most useful biomarkers to make the preliminary diagnosis through blood tests are: IgA anti-tissue transglutaminase (tTG), antiendomysium (EMA), and antideaminated forms of gliadin peptides (DGP) antibodies, since they have the highest accuracy [1,2]. Detection of $\operatorname{IgG}$ anti-tTG antibodies, although with less specificity for CD diagnosis, is also used and, is particularly important when the patient has IgA deficiency. Furthermore, these antibodies are also used to monitoring $\mathrm{CD}$ patients because its levels tend to decrease after 6 to 24 months on a gluten-free diet [3].

The improvement of blood tests efficacy to detect the CD could allow a comprehensive screening of the population and, thus, properly treat people suffering from this uncomfortable disease by implementing a gluten-free diet as soon as possible. However, the screening programs are not carried out because, currently, routine analyses are too expensive, and the administration cannot afford this cost for a broad spectrum of the population. For this reason, the development of low cost analytical devices that can be used in screening programs is a meaningful goal.

In recent years, enzyme linked immunosorbent assays (ELISA) have been the most widely used technique for the detection of biomarkers [4]. ELISA can be used to detect virtually any protein due to the potential manufacture of specific antibodies to the analyte of interest. However, this technique has certain drawbacks such as the need of bulky instrumentation and the possibility to get false signals from the colorimetric detection. Therefore, the electrochemical detection method seems to be a promising alternative in view of the equipment miniaturization, multianalyte detection capability and the requirement of low sample volume. Furthermore, large-scale production of these electronic devices allows a significant reduction in the cost. The adaptation of ELISA to the electrochemical detection is the electrochemical immunosensors (EIs).

EIs are self-contained integrated devices capable to provide quantitative analytical information using an immobilized immunological recognition element and an electrochemical based-transducer which converts the biological interaction into a measureable signal [5]. Ideal characteristics of EIs are: low cost and disposable devices, with inexpensive readers and with fast response without sacrificing good analytical characteristics for the resolution of the clinical problem of interest. Furthermore, they should be portable to be used in a decentralized way and by non-technical users at home, an ambulance or health centres. These are called point-of-care (POC) devices [6], defined as medical testing at or near the site of patient care, often accomplished through the use of handheld instruments. The most representative example is the glucose sensor for diabetics introduced in the last decades of the twentieth century.

\section{Electrochemical Immunosensors for the Detection of Celiac Disease Biomarkers}

Regarding the clinical diagnosis of CD using EIs, some advances are being achieved. Several methodologies and electrochemical devices have been developed in recent years for the detection of serological biomarkers of celiac disease.

One of the first EIs for the detection of anti-tTG autoantibodies was reported by Balkenhohl et al. [7] After the immobilization of tTG on the surface of screen-printed gold electrodes, a sandwich assay was carried out with the sample and an antibody labelled with horseradish peroxidase (HRP). Then, an enzymatic reaction resulting 
in a precipitated product was performed, which increases the charge transfer resistance (Rct) proportionally to the amount of biomarker in the sample. The change in Rct is measured by electrochemical impedance spectroscopy. Another EI developed by Pividori et al. was performed after physical adsorption of tTG on graphite-epoxy composite (GEC) electrodes. [8] This system followed a sandwich assay similar to the previously described using HRP secondary antibody labelled, but using hydroquinone and amperometric detection. For serum samples a sensitivity of $70 \%$ and a specificity of $100 \%$ were achieved as compared with the commercial ELISA method. Other electrochemical immunosensor for the detection of human anti-tTG antibodies was developed by Dulay et al. [9] In this case, gold disk electrodes were used as transducers and were modified with selfassembled monolayers to covalently bound tTG. After the immunological reaction using HRP labelled secondary antibodies, amperometric detection using TMB as enzymatic substrate was performed, obtaining a clinically relevant dynamic linear range and detection limit. A possible advantage of the covalent immobilization may be the largest capacity for the immunoreaction and lower nonspecific adsorption.

Meanwhile, Adornetto et al. developed a magnetoelectrochemical immunosensor for the detection of anti-tTG antibodies [10]. This system uses magnetic beads modified with tTG to perform a sandwich assay using secondary antibodies labelled with alkaline phosphatase. $\alpha$-naphtyl phosphate is used as enzymatic substrate and naphtol is generated as electroactive product. The detection is carried out subsequently using screenprinted electrodes with the aid of a magnet to preconcentrate the magnetic beads. A similar system using GEC electrodes as electrodic platform and peroxidase as label was developed by Kergaravat et al [11].

A disposable EI developed with screen-printed electrodes for the detection of IgA and IgG anti-tTG autoantibodies was developed by Neves et al. [12] Screen-printed carbon electrodes were modified with nanohybrid structures (gold nanoparticles/carbon nanotubes) and tTG was immobilized by simple adsorption on the nanostructured electrode surface, generating a high-stability immunosensor. The analytical signal was based on the anodic redissolution of enzymatically-generated silver by cyclic voltammetry. This system exhibited excellent characteristics and led to the amplification of the immunological interaction. The results obtained with the EI developed were successfully corroborated with ELISA tests. A similar platform but using $\mathrm{CdSe} / \mathrm{ZnS}$ quantum dots (QDs) labelled secondary antibodies for the detection of anti-tTG IgG antibodies was carried out by Martín-Yerga et al [13]. In this case, the detection of $\mathrm{Cd}$ (II) released from the QDs after an acid attack was proportional to the immunological reaction. Employing a direct label allows to save time analysis and reagents cost by eliminating the enzymatic reaction. In addition, for the fabrication of the sensor, 8-channel screen-printed carbon electrochemical arrays were used as transducers, being possible to perform 8 simultaneous analyses without an increment in time.

It is noteworthy, the first EI developed for the detection of antibodies against deaminated gliadin peptides (DGP) reported by Neves et al. [14] Following a similar methodology to the previously described by the same authors, nanohybrid screen-printed electrodes were modified by adsorption with DGP generating the sensor surface. Real serum samples were successfully assayed and the results were corroborated with an ELISA kit. The high specificity of DGP antibodies makes the presented EI a promising analytical tool for celiac disease diagnosis. Moreover, the same authors reported a multiplexed immunosensor using nanostructured screen-printed electrodes with two working electrodes modified with gliadin and tTG for the simultaneous detection of its specific autoantibodies (IgA and $\operatorname{IgG}$ ) in real samples [15]. The proposed sensor is a sensitive and effective methodology to the simultaneous determination of $\operatorname{IgA}$ and $\mathrm{IgG}$ antibodies against gliadin and tissue-transglutaminase.

In relation to the detection of gliadin autoantibodies, although being a less specific biomarker for CD diagnosis by serological tests, some electrochemical immunosensors for the detection of antigliadin antibodies have been reported. For instance, Rosales et al. developed an EI using gold electrodes with oriented covalent modification of gliadin on the electrode surface [16]. The amperometric detection was also conducted using the enzymatic system HRP-TMB. A simpler methodology developed by Neves et al. (explained previously) using nanostructured screenprinted was also used for the detection of anti-gliadin antibodies (IgA, IgG) in human real samples [17]. The obtained results were supported with a commercial ELISA test.

In summary, considering the electrochemical immunosensors reported for the detection of $\mathrm{CD}$ biomarkers, we would like to highlight the work carried out by Neves et al. The use of low-cost nanostructured screen-printed electrodes as immunosensor platform, simple experimental procedure, reduced sample volume and a very sensitive and fast voltammetric detection, place these devices as the preferred for the manufacture of POC devices for the detection of celiac disease. Furthermore, these EIs have shown to operate successfully with different celiac disease biomarkers such as anti-gliadin, anti-tTG and anti-DGP antibodies and in a multiplexed system.

\section{Future Perspectives}

Certainly, electrochemical immunosensors are still not in place to carry out the diagnosis of diseases in hospitals or medical centres. Some reasons could include the complex automatization of such devices, the sophisticated instrumentation required for multianalysis and the longterm stability of the sensing elements. However, a large scientific community is working towards the development of analytical diagnostic devices and those issues may soon be solved. On the one hand, it is already possible to perform multianalyte detection and simultaneous analyses with multichannel electrochemical devices, proving the utility for medical facilities where many analyses are carried out. On the other hand, this technology allows the fabrication of low-cost point-of-care devices that could be used ultimately for patients themselves, such as the glucose sensors widely employed bydiabetics. Definitely, celiac disease will also be diagnosed with point-of-care electrochemical devices in the coming years, sooner than expected. 


\section{References}

[1] A. Monzani, A. Rapa, P. Fonio, E. Tognato, L. Panigati, G. Oderda, Use of deamidated gliadin peptide antibodies to monitor diet compliance in childhood celiac disease., J. Pediatr. Gastroenterol. Nutr. 53 (2011) 55-60.

[2] S. Husby, S. Koletzko, I.R. Korponay-Szabó, M.L. Mearin, A Phillips, R. Shamir, et al., European Society for Pediatric Gastroenterology, Hepatology, and Nutrition guidelines for the diagnosis of coeliac disease., J. Pediatr. Gastroenterol. Nutr. 54 (2012) 136-60.

[3] M. Vives-Pi, S. Takasawa, I. Pujol-Autonell, R. Planas, E. Cabre, I. Ojanguren, et al., Biomarkers for diagnosis and monitoring of celiac disease., J. Clin. Gastroenterol. 47 (2013) 308-13.

[4] R.M. Lequin, Enzyme immunoassay (EIA)/enzyme-linked immunosorbent assay (ELISA), Clin. Chem. 51 (2005) 2415-8.

[5] S. Rodriguez-Mozaz, M.J. Lopez de Alda, D. Barceló, Biosensors as useful tools for environmental analysis and monitoring., Anal. Bioanal. Chem. 386 (2006) 1025-41.

[6] V. Gubala, L.F. Harris, A.J. Ricco, M.X. Tan, D.E. Williams, Point of care diagnostics: status and future., Anal. Chem. 84 (2012) 487-515.

[7] T. Balkenhohl, F. Lisdat, Screen-printed electrodes as impedimetric immunosensors for the detection of antitransglutaminase antibodies in human sera., Anal. Chim. Acta. 597 (2007) 50-7.

[8] M.I. Pividori, a Lermo, a Bonanni, S. Alegret, M. del Valle, Electrochemical immunosensor for the diagnosis of celiac disease., Anal. Biochem. 388 (2009) 229-34

[9] S. Dulay, P. Lozano-Sánchez, E. Iwuoha, I. Katakis, C.K. O'Sullivan, Electrochemical detection of celiac disease-related anti-tissue transglutaminase antibodies using thiol based surface chemistry., Biosens. Bioelectron. 26 (2011) 3852-3856.

[10] G. Adornetto, G. Volpe, A. De Stefano, S. Martini, G. Gallucci, A. Manzoni, et al., An ELIME assay for the rapid diagnosis of coeliac disease., Anal. Bioanal. Chem. 403 (2012) 1191-4.

[11] S. V Kergaravat, L. Beltramino, N. Garnero, L. Trotta, M. Wagener, M. Isabel Pividori, et al., Electrochemical magneto immunosensor for the detection of anti-TG2 antibody in celiac disease., Biosens. Bioelectron. 48 (2013) 203-9.

[12] M.M.P.S. Neves, M.B. González-García, H.P. a Nouws, A. CostaGarcía, Celiac disease detection using a transglutaminase electrochemical immunosensor fabricated on nanohybrid screenprinted carbon electrodes., Biosens. Bioelectron. 31 (2012) 95-100

[13] D. Martín-Yerga, M.B. González-García, A. Costa-García, Electrochemical immunosensor for anti-tissue transglutaminase antibodies based on the in situ detection of quantum dots., Talanta. 130 (2014) 598-602.

[14] M.M.P.S. Neves, M.B. González-García, H.P. a Nouws, A. CostaGarcía, An electrochemical deamidated gliadin antibody immunosensor for celiac disease clinical diagnosis., Analyst. 138 (2013) 1956-8.

[15] M.M.P.S. Neves, M.B. González-García, C. Delerue-Matos, A Costa-García, Multiplexed electrochemical immunosensor for detection of celiac disease serological markers, Sens. Actuators B. 187 (2013) 33-39.

[16] L.C. Rosales-Rivera, J.L. Acero-Sánchez, P. Lozano-Sánchez, I. Katakis, C.K. O'Sullivan, Electrochemical immunosensor detection of antigliadin antibodies from real human serum., Biosens. Bioelectron. 26 (2011) 4471-6.

[17] M.M.P.S. Neves, M.B. González-García, A. Santos-Silva, A. Costa-García, Voltammetric immunosensor for the diagnosis of celiac disease based on the quantification of anti-gliadin antibodies, Sens. Actuators B. 163 (2012) 253-259. 